\title{
Mueller matrix method for determining morphology of polymer dispersed liquid crystal
}

\author{
Maksimyak A.P., Maksimyak P.P. and Negrych A.L. \\ Correlation Optics Department, Chernivtsi National University \\ 2 Kotsyubinsky St., 58012 Chernivtsi, Ukraine E-mail: nehrych@itf.cv.ua
}

Received: 10.11 .2009

\begin{abstract}
This work presents a new method for determining morphology of polymer dispersed liquid crystals based on the approach of Mueller matrices. The method allows monitoring separately both liquid crystal and polymer composite components.
\end{abstract}

Keywords: PDLC, morphology, liquid crystal, Mueller matrix.

PACS: 42.25.Ja, 42.30.Va, 42.70.Df, 42.79.Kr.

UDC: $535.51,535.012$

\section{Introduction}

Rapid development of optoelectronics and information technologies requires creation of new composite materials based on liquid crystals (LC). An example of such materials is polymer dispersed liquid crystals (PDLCs). The content of LC in these composites is more than 20\% [1]. The PDLC combines optical properties typical for the LCs with such general properties of polymer matrices as plasticity, flexibility, lack of fluidity, and reliability. In addition, PDLCs are well governed by electric fields and are used, e.g., in displays [2, 3], windows with controlled transparency [2, 4], light shutters [2, 5] and systems including controlled microlenses for coupling radiation into fibres [2, 5]. Widespread utilisation of PDLC composites requires examination of their morphology, in particular the shapes and sizes of anisotropic LC droplets and isotropic polymer part.

Nowadays the morphology of LC-polymer composites is determined using polarisation and scanning electron microscopies [6], as well as angular distribution measurements of the scattered light intensity. Optical techniques for investigating PDLCs have been revealed to by promising [7]. The main attention in frame of these techniques is paid to the effects of scattering and depolarisation of optical radiation, which depend on the incident light wavelength, the voltage applied to PDLC samples, and some features of fabrication of samples under test (e.g., a sort of LC and polymer, a percentage of LC in the polymer matrix, conditions for photopolymerisation, etc.) [8]. In this work we suggest a Muellermatrix approach for determining the PDLC morphology.

Ukr. J. Phys. Opt. 2010, V11, №1 


\section{Preparation of PDLC}

We used a polymer NOA 65 (Norland Inc., USA) with dispersed droplets of the LC referred to as E7 (Merck KGaA, Germany). The corresponding refractive indices for the light wavelength of $\lambda=589 \mathrm{~nm}$ were equal to $n_{o}=1.5216$ and $n_{e}=1.7462$, whereas the refractive index of the polymer was $n_{p}=1.524[7,8]$.

The PDLC was located between two glass plates with transparent electroconductive indium-tin oxide layer. The thickness of PDLC aligned between the plates was controlled by spacers and equalled to 7-20 $\mu \mathrm{m}$. We had fabricated PDLC cells with different sizes of LC droplets arranged randomly and the cells with the same sizes of LC droplets in a strictly ordered arrangement. In order to fabricate PDLC cells, we had applied the following technique. The $\mathrm{LC}$ was mixed with the polymer according to the weight ratio 1:1 for the cells with the ordered arrangement of the LC droplets and 1:4 for the cells with the random one. Then the mixture was stirred during a period of $30 \mathrm{~min}$ by a mechanical mixer. Air bubbles were pumped out by a compressor.

We radiated the cell by a mercury lamp (DRSh-250) for $20 \mathrm{~min}$ to obtain PDLCs with random sizes and random coordinate distributions of the LC droplets. For example, a scheme depicted in Fig. 1 was utilised for obtaining PDLCs with the ordered arrangement of LC droplets.

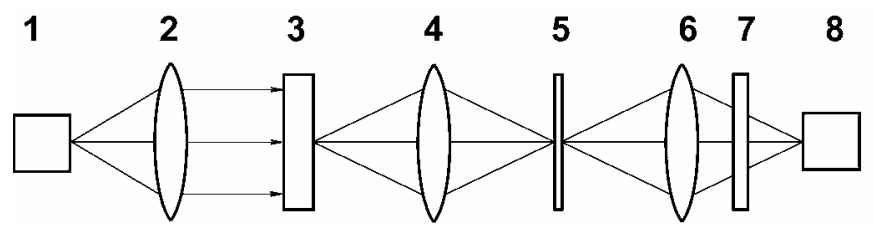

Fig. 1. Optical scheme used for photopolymerisation of PDLC: 1 - mercury lamp, 2 - collimator, 3 - photomask, 4, 6 - objectives, 5 - cell, 7 - optical filter, and 8 - CCD camera.

The mercury lamp DRSh-250 served as a source of ultraviolet radiation, with the power density of $50 \mathrm{~mW} / \mathrm{cm}^{2}$. A collimator 2 formed a parallel beam that illuminated a photomask 3. Image of the latter was projected to a plane 5 of PDLC cell with the aid of an objective 4 . An objective 6 and a CCD camera 8 allowed checking the image of photomask and so the accuracy of PDLC alignment. The photomask (see Fig. 2) was obtained by projecting a combination of regular hexagons with the sizes of $160 \mu \mathrm{m}$ onto a photographic film with the size of $2 \times 2 \mathrm{~cm}^{2}$. Under these conditions the LC droplets dispersed in the polymer matrix had the sizes of $50 \mu \mathrm{m}$.

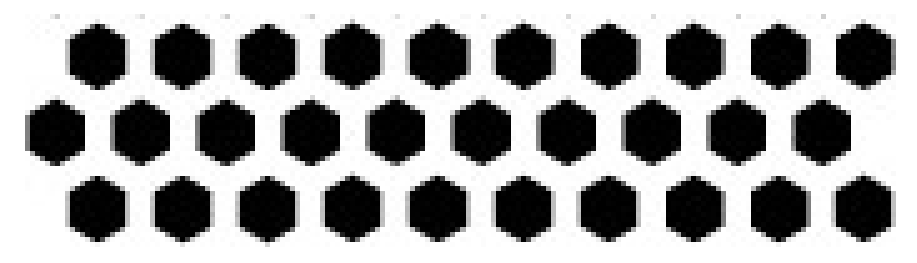

Fig. 2. Fragment of a photomask. 
Polarisation images of PDLCs with the ordered arrangement of the LC droplets are shown in Fig. 3. There is no clear allocation of isotropic and anisotropic parts on those images. Therefore we have suggested a visualisation technique aimed at determination of isotropic and anisotropic components and based on the use of Mueller matrices.

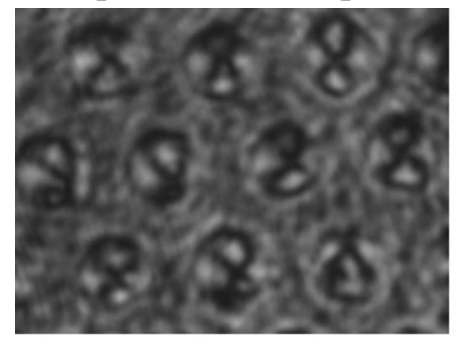

a

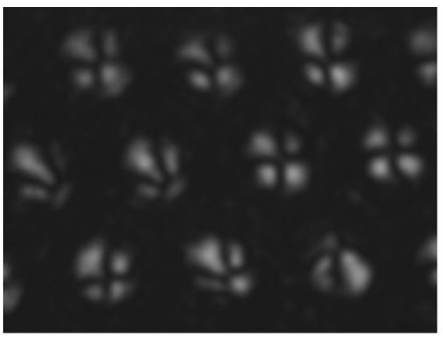

b

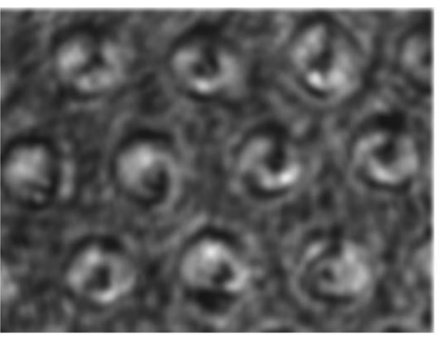

C

Fig. 3. Polarisation images of our PDLCs: (a) coaxial polarisers, (b) crossed polarisers, and (c) sum of the images (a) and (b).

\section{Mueller-matrix approach}

PDLCs consist of an optically active component (LC droplets) and an isotropic component (a polymer matrix). The matrix operator describing the properties of anisotropic medium is unified and constant in its structure. The Mueller matrix for each point of the LC component contained in the PDLC is described in the following way [9]:

$$
\{F\}=\left|\begin{array}{cccc}
1 & 0 & 0 & 0 \\
0 & \cos ^{2} 2 \theta+\sin ^{2} 2 \theta \cdot \cos \delta & \cos 2 \theta \sin 2 \theta(1-\cos \delta) & -\sin 2 \theta \sin \delta \\
0 & \cos 2 \theta \sin 2 \theta(1-\cos \delta) & \sin ^{2} 2 \theta+\cos ^{2} 2 \theta \cos \delta & \cos 2 \theta \sin \delta \\
0 & \sin 2 \theta \sin \delta & -\cos 2 \theta \sin \delta & \cos \delta
\end{array}\right|,
$$

where $\theta$ describes orientation of the optic axis and $\delta$ is the phase shift brought about by LC between the orthogonal components of a light wave amplitude. The Mueller matrix of the isotropic medium has the following form [9]:

$$
\{F\}=\left|\begin{array}{llll}
1 & 0 & 0 & 0 \\
0 & 1 & 0 & 0 \\
0 & 0 & 1 & 0 \\
0 & 0 & 0 & 1
\end{array}\right|
$$

One can see from Eqs. (1) and (2) that the off-diagonal elements of the Mueller matrix are equal to zero for the isotropic medium, while the matrix elements of the anisotropic medium may acquire arbitrary values.

We have set a problem that consists in finding such a combination of nonzero offdiagonal elements of the matrix $\{F\}$, which would not depend on the orientation $\theta$ of the optic axis and on the phase shift value $\delta$. In this case the Mueller matrix would visualise the anisotropic component, since the above combination of elements for the isotropic component remains to be zero. On the contrary, a combination of diagonal elements equal to zero would visualise the isotropic component of PDLC. 
In other words, if the combination of off-diagonal elements of the Mueller matrix responsible for anisotropic part is nonzero, we will be able to observe it against the background of zero contrast of polarization image of the combination of matrix elements for the isotropic component. Hence, we have a method for determination of the anisotropic component. If the combination of diagonal elements for the anisotropic part is zero, we will observe the isotropic part with a high contrast. In this way one can determine the isotropic component. The both methods are described below.

\subsection{The method for determination of anisotropic component}

Let us consider the sum of squares of the off-diagonal Mueller matrix elements $f_{24}, f_{42}, f_{34}$ and $f_{43}$ :

$$
\begin{array}{r}
f_{24}^{2}+f_{42}^{2}+f_{34}^{2}+f_{43}^{2}=2 f_{24}^{2}+2 f_{34}^{2}= \\
2 \sin ^{2} 2 \theta \sin ^{2} \delta+2 \cos ^{2} 2 \theta \sin ^{2} \delta=2 \sin ^{2} \delta
\end{array} .
$$

As seen from Eq. (3), the sum does not depend on the orientation $\theta$ of optic axis of the anisotropic component. Nonetheless, we will have to get rid of the phase shift $\delta$ between the orthogonal components of the wave amplitude in Eq. (3). It may be performed with the aid of variation of electric field applied to PDLC cell. Variation of the phase shift over one period enables us to exclude a dependence on $\delta$ involved in Eq. (3). Actually, integrating of Eq. (3) in the limits $[0 ; 2 \pi]$ gives

$$
\int_{0}^{2 \pi} 2 \sin ^{2} \delta d \delta=2\left(\frac{\delta}{2}-\left.\frac{\sin 2 \delta}{4}\right|_{0} ^{2 \pi}\right)=2 \pi .
$$

Thus, the definite integral of the sum of squares of the considered combination of offdiagonal Mueller matrix elements does not depend on $\theta$ and $\delta$ and is greater than zero.

\subsection{The method for determination of isotropic component}

As seen from Eq. (1), the Mueller matrix element $f_{44}$ is independent of the optic axis orientation $\theta$ and is nonzero. For the same reasons as for the method for determining the anisotropic phase, let us get rid of $\delta$ by integrating in the limits $[0 ; 2 \pi]$ :

$$
\int_{0}^{2 \pi} \cos \delta d \delta=\left(\left.\sin \delta\right|_{0} ^{2 \pi}\right)=0 .
$$

This means that the Mueller matrix element $f_{44}$ of the anisotropic component also equals to zero and so the isotropic component will be observed with a high contrast against its background.

\section{Experimental studies}

An optical scheme of our experimental setup is represented in Fig. 4. The light from a He-Ne laser 1 passes through a collimator 2 and a quarter-wave plate 3 and then trans- 
forms from linearly polarised into circularly polarised wave. Using a rotating polariser 4 and a quarter-wave plate 5 , we have implemented six different polarisations: $0^{\circ}, 45^{\circ}, 90^{\circ}$ and $135^{\circ}$, as well as left- and right-handed circular ones. The image of a PDLC cell 6 is formed by an objective 7 in a plane of CCD camera 10 and enters into a computer 11 . Analysis of the polarisations is performed with a quarter-wave plate 8 and a polariser 9 . All the measurements using this Stokes-polarimeter are automated and controlled by the computer.

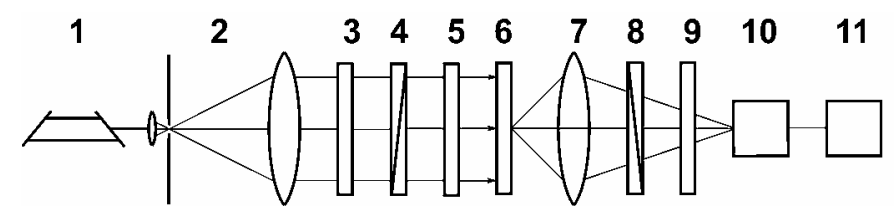

Fig. 4. Optical scheme of Stokes polarimeter: $1-\mathrm{He}-\mathrm{Ne}$ laser, 2 - collimator, 3,5,8- quarter-wave plates, 4, 9 - polarisers, 6 - PDLC sample, 7 - objective, 10 - CCD camera, and 11 - computer.

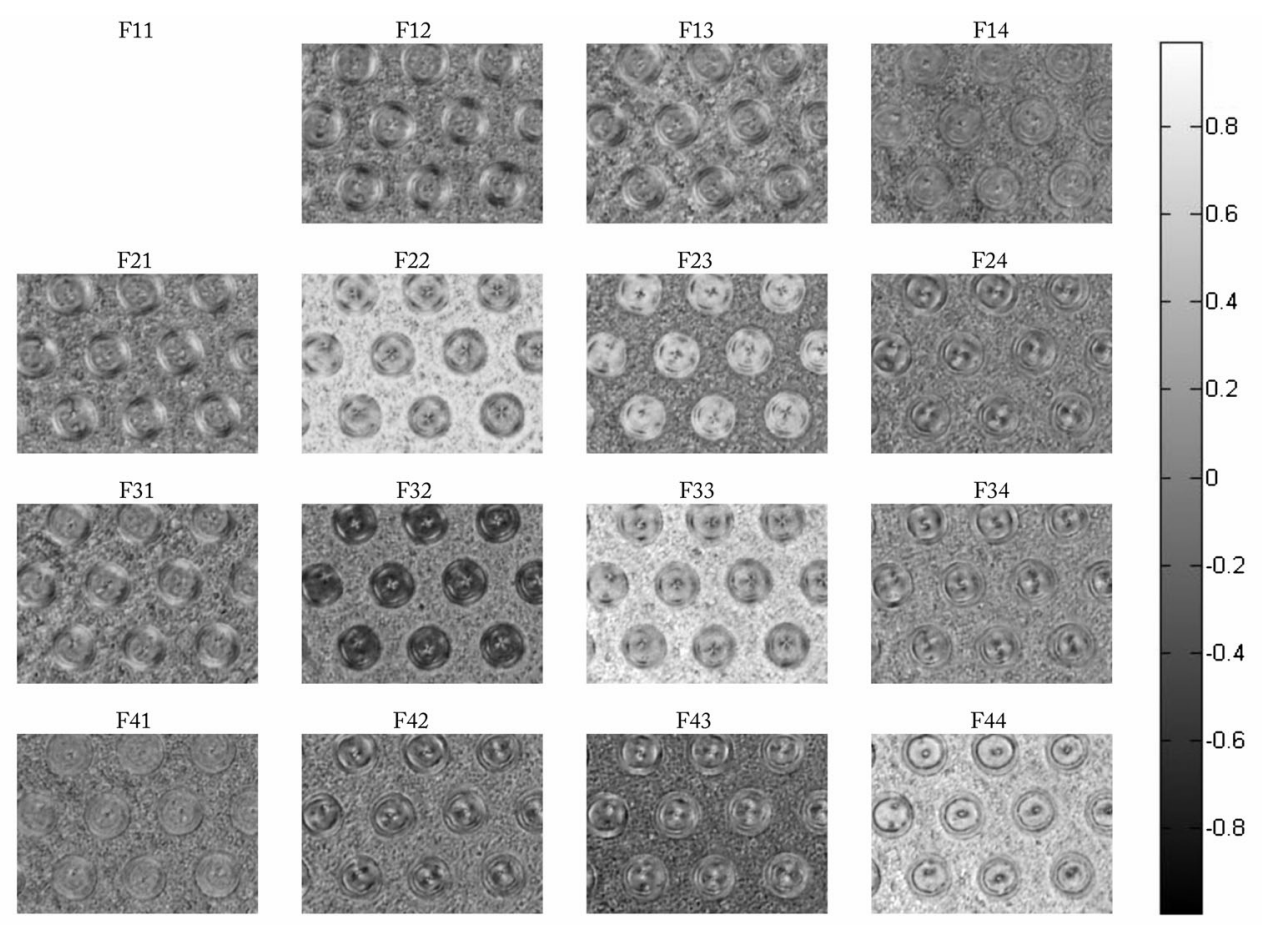

Fig. 5. Representation of Mueller matrix elements for one of PDLC images.

Using a set of 36 polarisation images obtained experimentally, we have calculated the Mueller matrix elements with the technique described in the monograph [9]. The resolution of our CCD camera is $640 \times 480$ and therefore we have calculated the Mueller matrix elements for each pixel. For diminishing experimental inaccuracies, the intensity corresponding to the noise of CCD camera has been subtracted from the intensity at each pixel. This has been fixed by dividing every (ij)-pixel of the Mueller matrix element by (ij)-pixel of the $f_{11}$ element. 


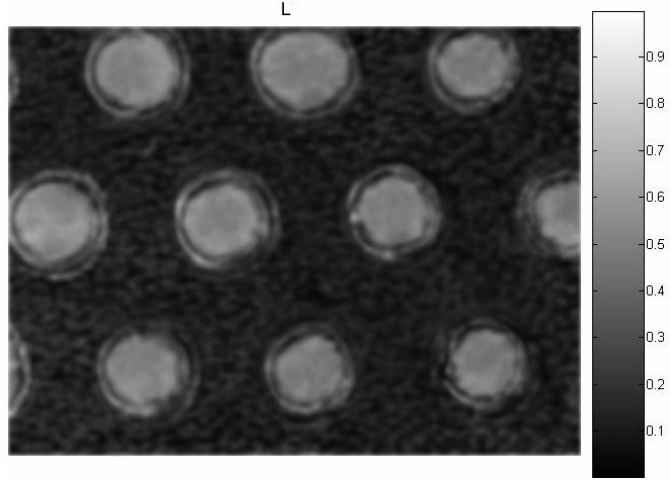

Fig. 6. Image of anisotropic part of PDLC with the regular alignment of LC droplets.

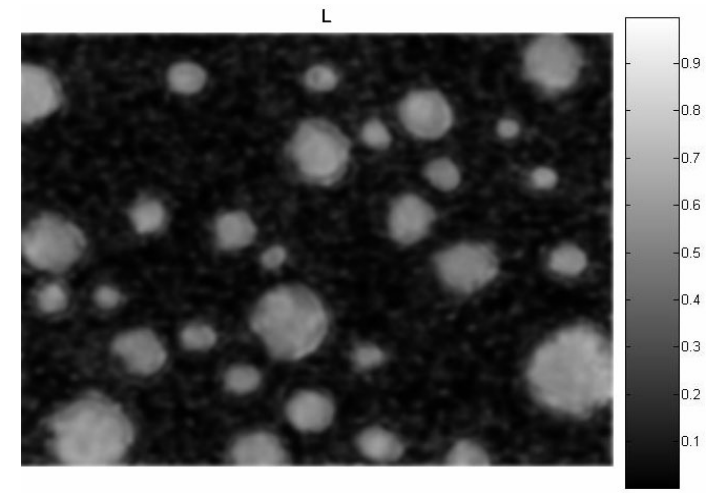

Fig. 8. Image of anisotropic part of PDLC with the random alignment of LC droplets.

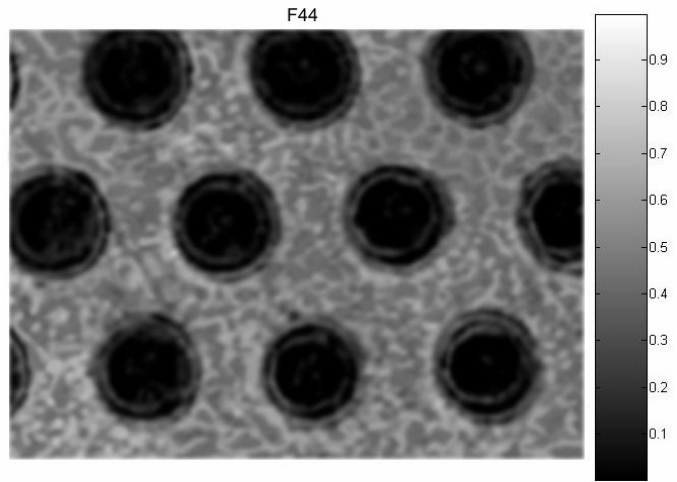

Fig. 7. Image of isotropic part of PDLC with the regular alignment of LC droplets.

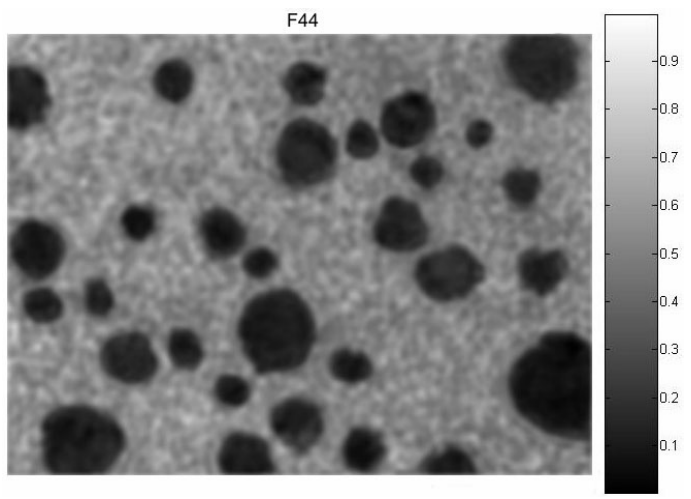

Fig. 9. Image of isotropic part of PDLC with the random alignment of LC droplets.

An example of calculated Mueller matrix elements for the image of PDLC having the ordered arrangement of LC droplets is shown in Fig. 5. It is easily seen that no clear image of the LC droplet is present in all of the figures.

Application of the method developed by us allows examining the PDLC morphology. To fulfil experimentally the algorithm given by Eqs. (4) and (5), we have replaced the integration over the period for summing:

$$
\int_{0}^{2 \pi} f_{i j} d f=\sum_{l=1}^{k} \frac{f_{i j}}{k},
$$

where $k$ is the amount of frames of CCD camera recording uniformly the phase shift variation by $2 \pi$. An ac voltage with the frequency of $f=1 \mathrm{kHz}$ has been applied to the PDLC layer. The operating range of the voltage has provided the phase shift variation of $2 \pi$ for the orthogonal polarisation components propagating in the LC. Thus we have calculated 16 arrays of Mueller matrix elements for the PDLC images for each voltage within this range. Then summing of pixels of the Mueller matrix elements has been per- 
formed for those voltages. As a result, 16 arrays of the Mueller matrix elements for the LC images integrated over the phase difference in the limits of $2 \pi$ have been obtained.

A result obtained when summing the squares of the integrated elements $f_{24}, f_{42}, f_{34}$ and $f_{43}$ is shown in Fig. 6 for a sample with the ordered arrangement of LC droplets. A sufficiently clear image of the LC droplets is observed. The isotropic part of PDLC obtained from Eq. (5) is represented in Fig. 7. The method developed above is also applicable to PDLCs with random alignments of the LC droplets. The morphologies of both anisotropic and isotropic parts of the PDLC are represented in Fig. 8 and Fig. 9, respectively.

Finally, let us summarise in brief the main result of our studies. We have demonstrated the efficiency of Mueller matrix method applied for studying the morphology of PDLCs. A significant advance of our method is that the isotropic part of PDLC is visualised with a high contrast, irrespective of its inhomogeneity, orientation or optical thickness.

\section{References}

1. West J L, Doane J W and Zumer S, Liquid crystal display material comprising a liquid crystal dispersion in a thermoplastic resin. Pat. 4.685.771 US, G02F 1/13 (1987).

2. Doane J W, Liquid crystals - Applications and Uses. Singapore: World Scientific, (1990).

3. Tanaka K, Kato K, Tsuru S and Sakai S, 1994. Liquid Crystals Displays. J. Soc. Inf. Display. 2: 37-48.

4. Crawford G P. and Doane J W, 1992. Polymer Dispersed Liquid Crystals. Condensed Matter News. 1: 5-11.

5. West J L, Zhang K, Glushchenko A, Andrienko D, Tasinkevych M, and Reznikov Y, 2006. Colloidal particles at a nematic-isotropic interface: Effects of confinement. Eur.Phys.J.E 20: 237-246.

6. Dolgov L, Yaroshchuk O and Qiu L, 2007. SEM Investigations of the Polymer Morphology in the Liquid Crystal-Polymer Composites with Different Polymer Contents. Molecular Crystals and Liquid Crystals. 468: 335-344.

7. Bueno J M, Ozolinsh M and Ikaurieks G, 2008. Scattering and depolarization in a polymer dispersed liquid crystal cell. Ferroelectrics. 370: 18-28.

8. Ozolinsh M and Papelba G, 2004. Eye cataract simulation using polymer dispersed liquid crystal scattering obstacles. Ferroelectrics. 304: 207-212.

9. Gerrard A and Burch J M, Introduction to matrix methods in optics. New York: Wiley-Interscience Publication (1975).

Maksimyak A.P., Maksimyak P.P. and Negrych A.L., 2010. Mueller matrix method for determining morphology of polymer dispersed liquid crystal. Ukr.J.Phys.Opt. 11: 21-27.

Анотація. У даній роботі запропоновано новий метод для визначення морфології рідких кристалів диспергованих у полімері. Метод базується на формалізмі матриць Джсонса $i$ дозволяє окреме дослідження рідких кристалів і компонент полімерного композиту.

Ukr. J. Phys. Opt. 2010, V11, №1 